\title{
Essai d'évaluation du coût économique de la réalisation des cibles des objectifs du millénaire pour le développement liés à l'eau potable en Algérie
} An assessment essay of the economic cost of achieving the
targets of the Millennium Development Goals (MDGs) related
to drinking water in Algeria

\author{
Nabil Kherbache et Kamal Oukaci
}

Volume 30, numéro 2, 2017

Reçu le 25 août 2016, accepté le 5 avril 2017

URI : https://id.erudit.org/iderudit/1042924ar

DOI : https://doi.org/10.7202/1042924ar

Aller au sommaire du numéro

Éditeur(s)

Université du Québec - INRS-Eau, Terre et Environnement (INRS-ETE)

ISSN

1718-8598 (numérique)

Découvrir la revue

Citer cet article

Kherbache, N. \& Oukaci, K. (2017). Essai d'évaluation du coût économique de la réalisation des cibles des objectifs du millénaire pour le développement liés à l'eau potable en Algérie. Revue des sciences de l'eau / Journal of Water Science, 30(2), 157-169. https://doi.org/10.7202/1042924ar
Résumé de l'article

L'objectif de l'article est d'évaluer le coût économique de la réalisation de la cible 7.C des objectifs du Millénaire pour le développement (OMD) et de s'interroger sur les contraintes et les améliorations ressenties dans le service d'approvisionnement de l'eau potable en Algérie. L'approche méthodologique utilisée s'adosse sur l'analyse des documents, ouvrages, articles, données et informations relatives à l'investissement/financement du sous-secteur de l'eau potable en Algérie. Ces données nous ont été fournies par les directions centrales du ministère des Ressources en eau et de l'Environnement (MREE) et l'Algérienne des eaux (ADE). L'étude montre que l'évolution favorable des indicateurs de l'eau potable est due à une politique d'investissement massif dans ce sous-secteur. Le cumul des montants autorisés pour la période analysée (1999-2012) s'évalue à environ 23,459 milliards \$US dont seuls 61,6 \% ont été dépensés de manière effective. La faiblesse de la capacité d'absorption s'explique par des entraves lors de l'exécution des budgets alloués et un manque d'un capital intellectuel capable de relever effacement le défi des OMD inhérents à l'eau et de gérer durablement les ressources en eau. Ces efforts se sont heurtés à des contraintes sur le terrain en raison d'une concentration sur la mobilisation de l'eau en amont (politique de l'offre) sans valorisation de la ressource en aval par une gestion de la demande en eau (GDE). Toutefois, l'Algérie a pu réaliser les cibles des OMD liés à l'accès à l'eau, avant l'échéance fixée par l'ONU en 2015, avec un coût économique qui s'estime à 14,515 milliards \$US. Le pays assure un accès à l'eau potable à la majorité de la population avec un taux de raccordement de $98 \%$ en 2015 contre $78 \%$ en 1999 et il a pu atteindre l'objectif lié à l'assainissement où le taux de raccordement aux réseaux d'assainissement s'évaluait en 2015 de $90 \%$ alors qu'il était de 73,2 $\%$ en 2000. 


\section{ESSAI D'ÉVALUATION DU COÛT ÉCONOMIQUE DE LA RÉALISATION DES CIBLES DES OBJECTIFS DU MILLÉNAIRE POUR LE DÉVELOPPEMENT LIÉS À L'EAU POTABLE EN ALGÉRIE}

An assessment essay of the economic cost of achieving the targets of the Millennium Development Goals (MDGs) related to drinking water in Algeria

NABIL KHERBACHE*, KAMAL OUKACI

Université de Bejaia, Faculté des sciences économiques, commerciales et des sciences de gestion, Laboratoire Économie et Développement (LED), 06000 Bejaia, Algérie

Reçu le 25 août 2016, accepté le 5 avril 2017

\section{RÉSUMÉ}

L'objectif de l'article est d'évaluer le coût économique de la réalisation de la cible 7.C des objectifs du Millénaire pour le développement (OMD) et de s'interroger sur les contraintes et les améliorations ressenties dans le service d'approvisionnement de l'eau potable en Algérie. L'approche méthodologique utilisée s'adosse sur l'analyse des documents, ouvrages, articles, données et informations relatives à l'investissement/ financement du sous-secteur de l'eau potable en Algérie. Ces données nous ont été fournies par les directions centrales du ministère des Ressources en eau et de l'Environnement (MREE) et l'Algérienne des eaux (ADE). L'étude montre que l'évolution favorable des indicateurs de l'eau potable est due à une politique d'investissement massif dans ce sous-secteur. Le cumul des montants autorisés pour la période analysée (1999-2012) s'évalue à environ 23,459 milliards \$US dont seuls $61,6 \%$ ont été dépensés de manière effective. La faiblesse de la capacité d'absorption s'explique par des entraves lors de l'exécution des budgets alloués et un manque d'un capital intellectuel capable de relever effacement le défi des OMD inhérents à l'eau et de gérer durablement les ressources en eau. Ces efforts se sont heurtés à des contraintes sur le terrain en raison d'une concentration sur la mobilisation de l'eau en amont (politique de l'offre) sans valorisation de la ressource en aval par une gestion de la demande en eau (GDE). Toutefois, l'Algérie a pu réaliser les cibles des OMD liés à l'accès à l'eau, avant l'échéance fixée par l'ONU en 2015, avec un coût économique qui s'estime à 14,515 milliards \$US. Le pays assure un accès à l'eau potable à la majorité de la population avec un taux de raccordement de $98 \%$ en 2015 contre $78 \%$ en 1999 et il a pu atteindre l'objectif lié à l'assainissement où le taux de raccordement aux réseaux d'assainissement s'évaluait en 2015 de $90 \%$ alors qu'il était de $73,2 \%$ en 2000.

Mots-clés : Eau potable, OMD, financement, budget d'équipement, capacité d'absorption. 


\section{ABSTRACT}

This paper aims to assess the economic cost of achieving the target 7.C of the Millennium Development Goals (MDGs) and to discuss constraints and improvements in drinking water delivery services in Algeria. The methodological approach used is based on the analysis of documents, articles, data and information about investment/funding of drinking water subsector in Algeria. The data used were provided by the Ministry of Water Resources and Environment and the Algerian Water Company. Our study confirms that the favorable evolution in drinking water indicators is due to massive investment in this sub-sector. In fact, the overall amount authorized during the analyzed period (1999-2012) is valued at US\$23.459 billions, of which, only $61.6 \%$ were actually spent. The weakness of absorptive capacity is due to many constraints in the execution of the allocated budgets and a lack of an intellectual capital able to face the challenge of MDGs inherent to water and to manage the water resources sustainably. Efforts to meet such challenges have been hampered by many constraints owing to concentration on the water mobilization upstream (supply-side management) without a downstream valorization by a water demand management (WDM). However, Algeria has been able to achieve the targets of the MDGs related to the drinking water services before the UN fixed deadline in 2015, with an economic cost estimated at US\$14.515 billions. Indeed, Algeria ensured access to safe drinking water to the majority of the population with a connection rate of $98 \%$ in 2015 , compared to $78 \%$ in 1999 . Moreover, it achieved the sanitation facilities target with a connection rate to the sewerage network of $90 \%$ in 2015 compared to $73.2 \%$ in 2000 .

\section{Key Words: Drinking water, MDG, funding, capital expenditure, absorptive capacity.}

\section{INTRODUCTION}

Les objectifs du Millénaire pour le développement (OMD) : "sont des buts ambitieux et bien intentionnés dans des domaines choisis, souvent assortis d'une dimension quantitative (par exemple, réduire de moitié la faim dans le monde et pas simplement de la faire reculer) et de délais bien définis (tous les pays sont exhortés à atteindre tous les buts d'ici septembre 2015)"(BHAGWATI, 2010). La définition précédente montre que les OMD couvrent des domaines divers et choisis. L'accès à l'eau n'est cité explicitement que dans l'objectif 7 : «Préserver l'environnement", sous la cible 7.C : « réduire de moitié, d'ici à 2015, le pourcentage de la population qui n'a pas d'accès à un approvisionnement en eau potable ni à des services d'assainissement de base ». Cette dernière cible liée à l'assainissement a été intégrée lors du Sommet de la Terre de
2002 à Johannesburg. Nous pouvons remarquer aussi que les ressources en eau sont citées implicitement dans la cible 7.A : "Intégrer les principes du développement durable dans les politiques et programmes nationaux et inverser la tendance à la déperdition des ressources environnementales " (ASSEMBLÉE GÉNÉRALE DES NATIONS UNIES, 2000). Toutefois, il est d'ores et déjà reconnu que la réalisation de plusieurs autres objectifs passe par un approvisionnement sain en eau et un service d'assainissement de base. L'agriculture est le plus grand consommateur de l'eau avec $70 \%$ des prélèvements mondiaux, voire plus dans certains pays pauvres (WWDR4, 2012). En améliorant l'efficience d'utilisation de l'eau dans l'agriculture et la productivité de l'eau, les pays peuvent abaisser le taux de la pauvreté et réduire ainsi le pourcentage de population qui souffre de la faim. De même, en réduisant le temps de recherche de l'eau, les enfants auront du temps pour suivre une éducation primaire. Selon l'OMS un accès à une eau salubre peut réduire les taux de la mortalité infantile et lutter efficacement contre les maladies à transmission hydriques (MTH) et d'autres épidémies émergeant là où les systèmes d'assainissement sont absents. Donc, le lien entre l'accès à l'eau potable et les autres OMD est bien établi.

À cet effet, les pays s'efforcent à satisfaire la demande en eau potable de la population, mais fréquemment la volonté affichée bute sur une rareté des ressources en eau c'est l'exemple de l'Algérie qui se trouve dans une situation de rareté d'eau aigüe avec une dotation de $437 \mathrm{~m}^{3} \cdot \mathrm{an}^{-1}$ par habitant en 2012 . Les projections tablent sur un ratio de 320 et $300 \mathrm{~m}^{3} \cdot \mathrm{an}^{-1}$ par habitant en 2030 et 2050 respectivement. L'indice d'exploitation (Water Ressources Vulnerability Index [WRVI]) a atteint 87,1\% en 2012 (KHERBACHE, 2013, 2014a et 2014b; BROWN et MATLOCK, 2011; RIJSBERMAN, 2006). Laccroissement démographique et la population davantage urbanisée rendent l'accès au service de l'eau difficile et coûteux surtout pour les villes qui, compte tenu de l'exploitation totale des ressources proches, doivent recourir à la mobilisation de l'eau sur des distances de plus en plus lointaines. Dans ce contexte, des projets de transferts et d'interconnexions, sur des centaines de kilomètres, ont été réalisés. Parmi ces grands projets en Algérie, il y a le transfert In Salah-Tamanrasset (réceptionné en 2011, il consiste à prélever $100000 \mathrm{~m}^{3}$ par jour et la ramener depuis In Salah jusqu’à Tamanrasset sur une distance de plus de $750 \mathrm{~km}$ ); le transfert Koudiat Acerdoune (Bouira) vers Tizi Ouzou, Médéa et M'sila; le transfert des hautes plaines Sétifiennes; le transfert Mestghanem-Oran-Arzew (MOA); le barrage Beni Haroun, etc. Ces réalisations ont été accompagnées par un processus accéléré de réformes institutionnelles du secteur de l'eau notamment après la promulgation de la loi No 05-12 relative à l'eau. L'ensemble de ces efforts s'est heurté prématurément à des contraintes majeures sur le terrain en raison d'une concentration sur la mobilisation de l'eau en amont (politique de l'offre) sans valorisation de la ressource en aval par une gestion de la demande en eau (GDE). Toutefois, l'Algérie a pu 
réaliser les cibles des OMD liés à l'accès à l'eau bien avant leurs échéances fixées par l'ONU en 2015. De fait, le pays assure un accès à l'eau potable à la majorité de la population avec un taux de raccordement de $98 \%$ en 2015, contre $78 \%$ en 1999. Et elle a pu atteindre l'objectif lié à l'assainissement où le taux de raccordement aux réseaux d'assainissement s'évalue en 2015 de $90 \%$ alors qu'il était de 73,2\% en 2000.

Nous nous interrogeons dans cet article sur le coût économique de la réalisation de la cible 7.C. En fait, l'évolution favorable des indicateurs de l'eau potable est due à une politique d'investissement massif dans ce sous-secteur. Le cumul des montants autorisés pour la période analysée (1999-2012) s'évalue à environ 23,459 milliards \$US dont seuls 14,515 milliards \$US ont été dépensés de manière effective, soit un taux d'absorption de 61,6\%. Une situation qui s'explique principalement par des entraves lors de l'exécution des budgets alloués et un manque de capital intellectuel (humain) capable de relever effacement le défi des OMD inhérents à l'eau et ipso facto gérer durablement les ressources en eau. Le capital intellectuel est un concept forgé par TURTON (2002) pour désigner l'ensemble des compétences intervenant dans la gestion de la composante structurelle de la capacité d'adaptation à côté de la composante sociale. Il est structurant pour garantir une transition d'un modèle de l'offre (supply-side management) à la politique de GDE (water demand management ou demand-side management) et de la protection de l'eau.

\section{MÉTHODOLOGIE}

L'étude a été conduite en Algérie dans le but d'évaluer le coût économique des cibles des OMD liés à l'eau potable. Pour ce faire, notre approche méthodologique est multidimensionnelle. Elle s'adosse fondamentalement sur l'analyse des documents, données relatives à l'investissement/ financement du sous-secteur de l'eau potable en Algérie qui nous ont été fournies par les directions centrales du ministère des Ressources en eau et de l'Environnement (MREE). Il est à signaler qu'avant le remaniement gouvernemental partiel du 14 mai 2015, le ministère chargé des ressources en eau s'appelait : ministère des Ressources en eau (MRE). Le MREE est régi par les dispositions du décret exécutif No 16-89 du $1^{\text {er }}$ mars 2016 portant organisation de l'administration centrale du ministère des Ressources en eau et de l'Environnement. En outre, notre approche se base sur l'analyse des données du ministère des Finances algériennes (MF) et de l'Algérienne des eaux (ADE). Cet article a été élaboré aussi moyennant des discussions et entretiens avec les cadres et responsables de certains acteurs intervenant dans la mise en place de la politique nationale de l'eau potable. Cette évaluation du coût, pour la période allant de 1999 à 2012, abrite tous les programmes confondus tels qu'ils sont présentés dans les plans d'exécution annuelle du budget de l'équipement (dépenses d'investissement) du MREE. L'étude porte sur les programmes sectoriels centralisés (PSC), gérés par le MREE ou les établissements sous tutelle dotés d'une autonomie financière et les programmes sectoriels déconcentrés (PSD) gérés au niveau déconcentré, autrement dit à l'échelle de wilaya par les directions des Ressources en eau de wilaya (DREW). Quant aux plans communaux de développement (PCD) qui se gèrent à l'échelle infranationale (collectivités locales), ceux-ci entrent dans le cadre du budget du ministère de l'Intérieur et des collectivités locales. Notre évaluation de financement et du coût économique des améliorations dans le service de l'eau potable sera limitée, dès lors, aux deux premiers programmes en l'occurrence PSC et PSD. Les dépenses engagées ont été communiquées par le MREE et le MF en dinar algérien (DZD). Par conséquent, nous avons effectué des conversions (du DZD au \$US) en nous référant à une série de taux de change entre 1999-2012. Elle est tirée de la base de données de la banque mondiale. Cette série est calculée comme étant une moyenne annuelle sur la base des moyennes mensuelles qui fait référence au taux de change indiqué par la Banque d'Algérie. Létude mobilise aussi les statistiques sur les améliorations dans le service de l'eau potable collectées auprès des institutions chargées de la gestion de l'eau et nous avons utilisé intensivement les rapports d'étude d'actualisation du plan national de l'eau (PNE). Cet article se structure en trois grands axes. Tout d'abord, une présentation succincte des indicateurs du service de l'eau potable, les améliorations ressenties ainsi que certaines contraintes du service en Algérie sont exposées. Ensuite, l'analyse se porte sur les aspects qui touchent au poids du financement dans tout le secteur de l'eau en Algérie. Le sous-secteur de l'eau potable sera isolé dans l'étude en mettant l'accent sur le coût économique des améliorations enregistrées dans le service d'alimentation en eau potable (AEP) et de l'atteinte des cibles des OMD de l'eau via l'évaluation du coût dans le segment de l'AEP/adduction par une analyse rétrospective (1999-2012) et une analyse prospective à l'horizon 2030.

\section{RÉSULTATS ET DISCUSSION}

\subsection{L'eau potable en Algérie : indicateurs en améliorations et inégalités d'accès}

La région de l'Afrique du Nord y compris l'Algérie a atteint la cible liée à l'AEP bien avant son échéance. Le taux d'accès à l'eau pour la région de l'Afrique du Nord est estimé de $92 \%$ en 2012 contre $87 \%$ en 1990 (ONU, 2011, 2014a et 2014b). De même, la situation de l'accès à l'eau potable en Algérie a évolué positivement ces dernières années. 


\subsubsection{Le taux de raccordement aux réseaux publics d'AEP}

En dépit de l'évolution des taux de raccordement durant la période allant de 1966 à 2012, nous remarquons qu'en raison de l'accroissement démographique le nombre d'habitants non raccordés (en termes absolus) en 1998 (8,547 millions) est supérieur à celui de 1966 (7,554 millions) (HADJI, 2005) (Figure 1). Alors que le taux de non-raccordement en termes relatifs, autrement dit le nombre par rapport à la population totale de la période, est en baisse continue surtout pour la dernière décennie. Ce renversement de la tendance est la conséquence des réformes entamées depuis 2000, soit l'augmentation de la part du budget dédiée au secteur de l'eau (environ 4,77\% du PIB en 2009; 2,48 \% du PIB en 2012 contre 0,84 \% du PIB en 1999) et la réalisation des projets de transferts conséquents sur le service de l'eau potable. Cependant, ces données sont à prendre prudemment, car elles ne sont pas homogènes sur tout le territoire national. Elles dissimulent de facto une grande disparité régionale. À titre d'illustration, les données du MREE montrent qu’à la wilaya de Mostghanem le taux de raccordement ne dépasse pas $58 \%$ alors qu'à la wilaya de Bejaia et Blida le taux touche $99 \%$. L'accès à l'eau enregistre un écart important entre les zones urbaines et les zones rurales qui " restent très mal desservies ", selon la BANQUE MONDIALE (2007a). Ladite étude montre aussi que malgré le taux d'accès jugé satisfaisant dans les villes, celui des régions rurales demeure aux alentours de $89 \%$. D'ailleurs, il existe un décalage entre les estimations officielles et la réalité du terrain qui marque des écarts importants. L'évaluation officielle des taux de desserte des populations rurales aux réseaux d'AEP en 2007 était $82 \%$, or l'estimation réaliste table sur $80 \%$, il va de même pour l'accès à l'assainissement, officiellement $81 \%$ alors qu'en réalité ceci dépasse à peine $47 \%$ (BANQUE MONDIALE, 2007a et 2007b). En 2000, un écart important de $40 \%$ sépare le taux de raccordement à l'assainissement au niveau urbain et celui des régions rurales. Une tendance est vers l'absorption de cet écart, car en 1988, il était de $51 \%$ (ONU, 2005). Ces écarts portent atteinte à la gouvernance pluriniveaux (multilevel governance) et ils sont dus spécifiquement au déficit d'information (information gap) (OCDE, 2012; CHARBIT, 2011), à un manque de formation du capital intellectuel (TURTON, 2002) et à l'absence d'enquêtes de terrain au niveau national qui reflètent l'état réel de l'accès aux services de l'assainissement.

\subsubsection{Les fréquences de distribution et la qualité du service}

L'évaluation des quantités d'eau à mettre à la disposition des usagers se fixe souvent par les objectifs de dotation de l'eau par habitant. En revanche, la qualité s'étend sur deux volets, l'axe de l'eau proprement dite et celui de la qualité de service pour acheminer la ressource selon les besoins exprimés. Dans le contexte algérien, la qualité de service se mesure souvent par les fréquences de distribution. Or, d'autres indicateurs complémentaires et cruciaux pourraient être plus pertinents comme la dotation en eau par habitant, et ce sans incorporer les taux de pertes physiques dans les réseaux qui demeurent élevés, ou la réalisation d'enquêtes auprès des usagers notamment les ménages, car la qualité de service ne peut se mesurer qu'en degré de satisfaction des utilisateurs. En réalité, les enquêtes sont peu utilisées pour ne pas dire inutilisées, ce qui nous a amenés à prendre les estimations de l'ADE et des sociétés déléguées consolidées par les cadres de la direction de l'Alimentation en eau potable (DAEP) au niveau du MREE. Au premier abord nous remarquons une évolution favorable des fréquences de distribution de l'eau potable. Ainsi, la distribution quotidienne passe de 693 communes (45\%) en 1999 à 1156 communes en 2015 (75\%); à l'intérieur de ce taux, $38 \%$ des communes bénéficient d'un service de $24 \mathrm{~h} / 24$ et le reste bénéficie d'un service discontinu. Le MRE (2008) a exposé certains exemples d'amélioration comme la wilaya de Tlemcen (passée de 1 jour $/ 5$ au quotidien), d'Annaba (de 1 jour/2 au quotidien) et de Djelfa (de 1 jour $/ 5$ au quotidien). En dépit de ces améliorations, la distribution de l'eau se caractérise par des coupures et des politiques de rationnement horaire de la distribution qui engendrent souvent des augmentations de la consommation des ménages (KERTOUS, 2010). Lorsque le service de l'eau devient irrégulier, l'exploitation des moyens de stockage devient quant à lui un moyen pour faire face à la hantise de l'eau, dans ces conditions certains usagers possèdent des citernes et ils procèdent au stockage même dans les villes desservies $24 \mathrm{~h} / 24$, ce qui explique la perception sociale de la rareté de l'eau et la recherche des procédés d'adaptation individuelle à la rareté.

La qualité de l'eau distribuée est un autre axe de la qualité du service. Il constitue un point noir de la politique de l'eau potable en Algérie. La qualité de l'eau acheminée est une préoccupation majeure des abonnés qui sont réticents quant à la consommation de l'eau du robinet. Ceux-ci ont développé une capacité d'adaptation d'ordre individuel. À Bejaia par exemple, alors que les usagers sont réticents à payer une eau distribuée par l'ADE et fortement subventionnée, ils se trouvent contraints et forcés de rechercher les vendeurs d'eau qui sillonnent les quartiers et vendant une eau soi-disant de Toudja de meilleure qualité que celle livrée dans leurs robinets. Ils paient à cet effet le litre d'eau à $2 \mathrm{DZD}$ soit $2000 \mathrm{DZD} \cdot \mathrm{m}^{-3}$ $\left(27,17 \$ \mathrm{US} \cdot \mathrm{m}^{-3}\right)$, un prix exorbitant comparé au tarif administré appliqué jusqu'ici par les prestataires de service. En outre, les statistiques des institutions de l'eau prennent toujours le taux de raccordement comme indicateur d'évolution favorable du service de l'eau, mais à quoi sert d'avoir un raccordement au réseau et un robinet sans une ressource distribuée (ou distribuée de façon intermittente) à l'usager? Ainsi quelques quartiers riches d'Alger (Hydra, El-Mouradia) bénéficient de la distribution d'eau quotidiennement et avec une fréquence $24 \mathrm{~h} / 24$ souvent pour remplir des piscines, alors que d'autres quartiers moins aisés et populaires subissent des politiques de rationnement récurrentes et à grande échelle. Cette situation est amplifiée en raison de la non-application du mécanisme 


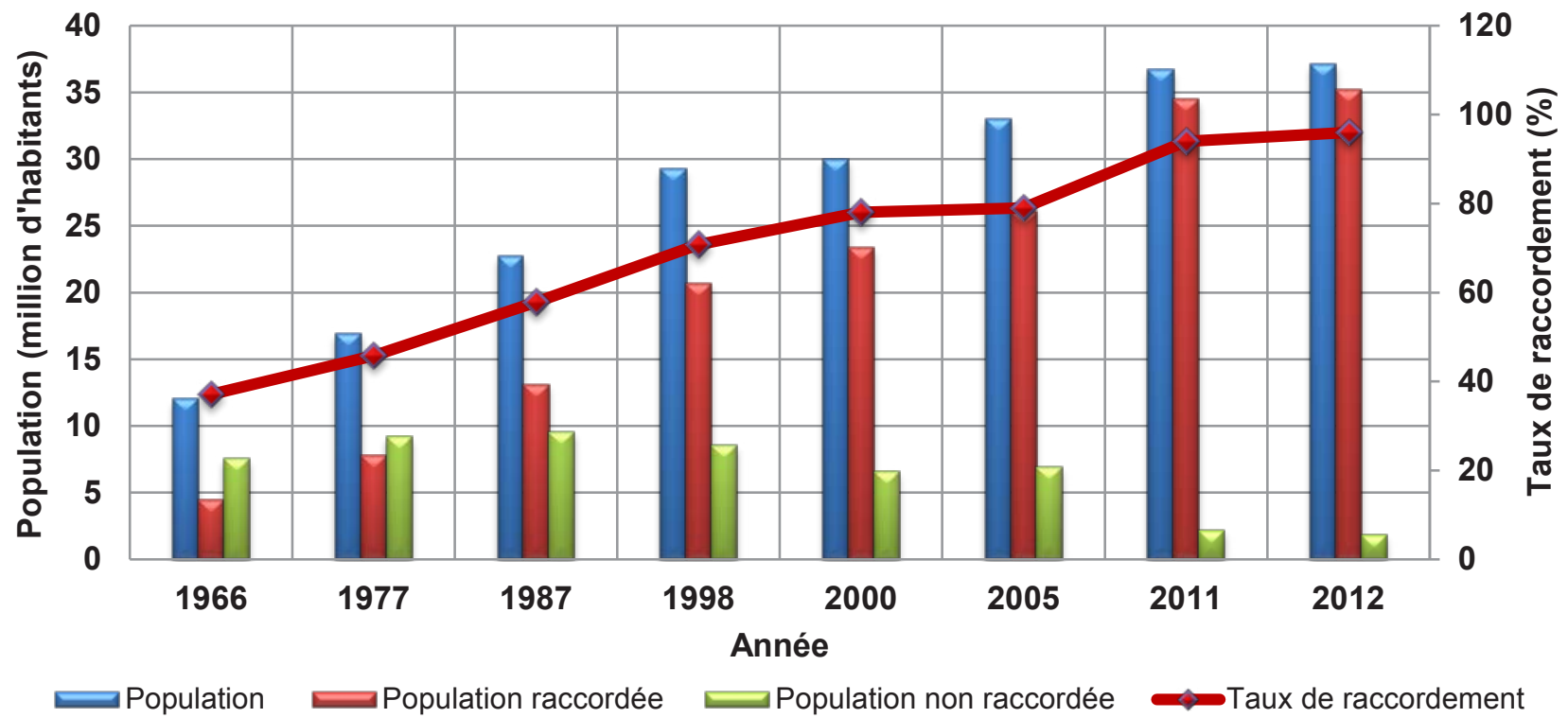

Figure 1. Évolution des raccordements à l'alimentation en eau potable (sources de données : Hadji, 2005; MRE, 2011). Evolution of the access rate to drinking water in Algeria.

de subvention croisée, mais aussi à cause du niveau de ces subventions qui profitent à tous les usagers et catégories (riches et pauvres) ce qui incite le gaspillage de cette ressource rare et précieuse.

\subsection{3 Évolution de la demande en eau potable et les dotations par tête}

En parallèle aux restructurations du secteur de l'eau potable et à la volonté affichée depuis 2000, l'Algérie vit durant cette même période une transition des indicateurs démographiques et elle table encore sur une continuité de ladite transition. Celle-ci est accompagnée d'une augmentation appréciable de la demande en eau potable suite à l'amélioration du niveau de vie algérien, mesuré en PIB par habitant. De fait, le PIB par tête est passé de 1 801,4 \$US en 2000 à 5 451,2 \$US en 2013. L'augmentation de la demande en eau potable s'explique aussi par l'urbanisation et une absence d'incitation à l'économie d'eau, soit par une tarification convenable ou par une véritable politique de l'eau. La transition de la demande en eau potable et avec elle de la dotation par habitant est illustrée à la figure 2 .

La demande en eau potable et la dotation sont en augmentation continue en Algérie. De fait, la demande a été multipliée par 2,5 soit un accroissement phénoménal de $150 \%$ entre 1999 et 2012. Elle est passée de $1,25 \mathrm{~km}^{3}$ en 1999 à $3,1 \mathrm{~km}^{3}$ en 2012. Selon les données du PNE, la demande en eau potable augmente de $8 \%$ par année. On remarque qu'il y a une corrélation positive entre cette demande, qui correspond en fait à la production de l'eau via les infrastructures de mobilisation, et le PIB per capita qui a connu un accroissement supérieur de 202,61 \% durant la même période (2000 et 2013). La même observation a été soulevée pour la dotation en eau par habitant; cette composante a connu aussi une augmentation égale à presque 42,5\% entre 1999 et 2012 . Toutefois, cette quantité ne concerne pas que les usagers domestiques parce que la demande en eau potable au sens du PNE regroupe la part des administrations, du commerce et de l'artisanat et des activités touristiques et, en plus, une part liée aux pertes dans les réseaux de distribution. Par conséquent, l'analyse de la composante de chaque volet pourrait abaisser la dotation par habitant à un niveau inférieur à $55 \mathrm{~L}$ par jour par habitant (MRE-GIZ, 2011). Ce qui demeure loin de la moyenne des pays développés ou ceux dont le niveau de développement est comparable avec l'Algérie.

Le goulot d'étranglement de ces améliorations se trouve dans la faiblesse de l'efficience de l'eau. L'efficience globale de l'eau potable pour l'ADE (2012) s'évalue de 41,37\% donc un taux de pertes (physiques et commerciales) de 58,63\%. Notons que ces pertes représentent $25,5 \%$ de la demande en eau potable de l'Algérie estimée de $3,1 \mathrm{~km}^{3}$ en 2012 (Figure 2). Autrement dit, sur $1,352 \mathrm{~km}^{3}$ d'eau produite par l'ADE en 2012 le volume facturé est estimé de $0,55933 \mathrm{~km}^{3}$ d'où un volume perdu de $0,792674 \mathrm{~km}^{3}$. Il importe de souligner que les pertes (commerciales et physiques) constituent aussi des pertes financières à l'établissement de distribution de l'eau. Pour cette raison l'absorption des pertes pourrait être source de recettes utilisables pour la couverture des coûts d'entretien et d'exploitation. Le volume perdu chez l'ADE est de $0,792674 \mathrm{~km}^{3}$ en 2012 , sur la base du tarif national moyen de $18 \mathrm{DZD} \cdot \mathrm{m}^{-3}$, les pertes financières s'articulent autour de 14,27 milliards DZD. Il va de soi que l'évaluation sur la base des coûts de mobilisation réels (sans subvention) de l'eau dévoile des pertes économiques et financières pour l'établissement de l'eau beaucoup plus importantes. Et l'aberration la plus 


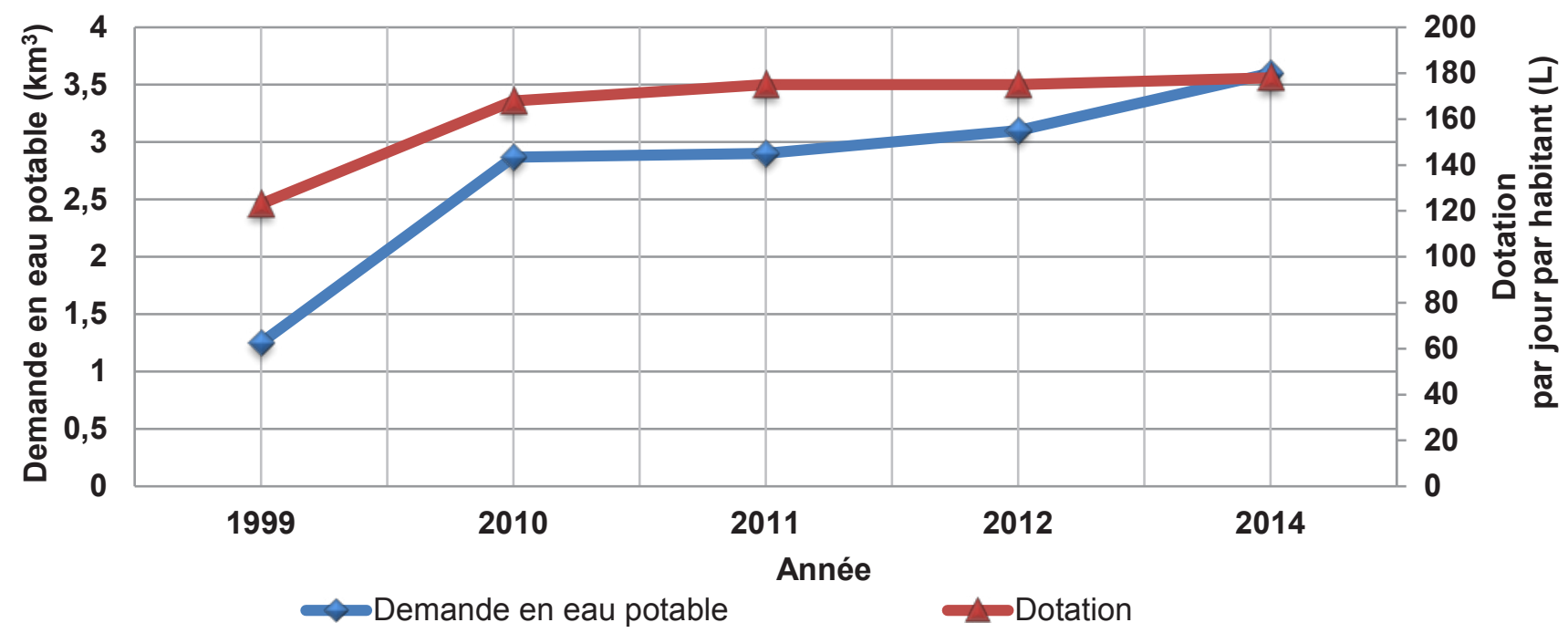

Figure 2. Évolution de la demande et de la dotation en eau potable en Algérie (source de certaines données : MRE, 2011). Evolution of drinking water demand and endowment in Algeria.

prégnante se trouve au niveau des stations de dessalement de l'eau de mer (KHERBACHE, 2014a). En 2012, le taux de rendement des réseaux (ou l'efficience globale de l'eau) est égal à $41,37 \%$ en moyenne ce qui veut dire que le taux de pertes et de fuites était 58,63\% (soit 792,674 $\mathrm{hm}^{3}$ ). Ce dernier est largement supérieur à la capacité nominale totale de dessalement de l'Algérie évaluée à $514,65 \mathrm{hm}^{3}$ en 2013. De fait, la production réelle de l'eau dessalée est aujourd'hui de 322,02 $\mathrm{hm}^{3}$ ce qui ne représente que 40,62\% de taux de pertes; ces chiffres confirment la propension des responsables à favoriser des politiques d'investissement plutôt que celles de l'exploitation. L'objectif d'absorption des pertes constitue un indicateur de la GDE. Le taux ne doit en aucun cas dépasser $20 \%$ (GUMBO, 2004). La GDE permettrait à l'Algérie une économie d'eau de $792 \mathrm{hm}^{3}$ par an, soit 25,5\% de la demande en eau potable.

Dès lors, les insuffisances sont nombreuses bien que le discours officiel confirme et affirme que le défi de l'eau a été parfaitement gagné et que les difficultés d'accès à l'eau ne se poseraient plus. Présentement, les habitants des communes et des régions éparses souffrent sévèrement de la hantise d'accès à l'eau salubre. À titre d'exemple, à Draà El Gaid, une commune qui se situe au sud-est de la wilaya de Bejaia abritant 30135 habitants sur une superficie de $123,34 \mathrm{~km}^{2}$ (DPSB, 2012), des enfants et des femmes sont livrés à la corvée de l'eau. Certains utilisent des procédés primitifs comme moyens d'approvisionnement en eau, et d'autres doivent attendre la livraison par camion-citerne détenu par les services de la commune d'où l'apparition des conflits d'usage. Le recours à la violence et à des moyens d'appropriation violents s'installe de manière aigüe quand une conjoncture de rareté ou de sécheresse se manifeste.

\subsection{Le financement du secteur de l'eau en Algérie : la primauté d'un secteur stratégique}

Cela fait déjà longtemps que les investissements dans le secteur de l'eau sont financés par concours définitif de l'État faute d'une participation de l'usager. En effet, le financement par emprunt des collectivités locales a été arrêté dans la fin des années soixante (CNES, 2000). L'Algérie a connu un véritable "plan Marshall de l'eau » depuis 1999, au point que d'aucuns annoncent que la bataille de l'eau a été bien gagnée, permettant ainsi de sortir de la rareté économique de l'eau (economic water scarcity) que l'Algérie a vécue durant des longues décades. Il est à signaler que le concept de la rareté économique de l'eau est un indicateur utilisé par l'Institut international de la gestion de l'eau (International Water Management Institute [IWMI]) pour mesurer la rareté de l'eau. RIJSBERMAN (2006) l'a mentionné dans son article : Water scarcity : Fact or fiction, afin de décrire les pays à forte dotation en eau renouvelable, mais faute d'une capacité d'investissement dans des infrastructures de mobilisation de l'eau, ils se voient en situation de pénurie d'eau.

De nos jours, le secteur de l'eau en Algérie décroche la part du lion dans tous les programmes de développement et d'équipement publics. Il y a lieu de noter que le financement des infrastructures et d'ouvrages de mobilisation de l'eau en Algérie se fait par le budget d'équipement de l'État. Celui-ci comporte deux éléments à savoir le budget d'investissement et les opérations en capital. Pour chaque programme spécifique de développement, un compte d'affectation spéciale (Special Treasury Accounts [STA]) est attaché. Nous avons distingué cinq STA qui participent, ou ont participé, au financement de l'eau en Algérie, que nous avons tiré de la nomenclature des comptes du trésor (2012) : 
- Le compte No 302-108 : "Compte de gestion des opérations d'investissements publics inscrites au titre du programme de soutien et de relance économique (PSRE) (2001-2004) " avec une enveloppe allouée au secteur de l'eau de 4,386 milliards DZD (soit 57,52 millions \$US).

- Le compte No 302-120 : "Compte de gestion des opérations d'investissements publics inscrites au titre du programme complémentaire de soutien à la croissance (PCSC 2005-2009) ». Ce compte a géré plus de $13 \%$ des ressources allouées au secteur de l'eau de ce programme soit 1820 milliards DZD (environ 25,92 milliards \$US).

- Le compte No 302-116 : "Fonds spécial pour le développement économique des hauts plateaux (FSDRHP) » avec une enveloppe de 43,2 milliards DZD (594,87 millions \$US) dédiée au secteur.

- Le compte No 302-089 : «Fonds spécial de développement des régions du Sud (FSDRS) » avec un montant du secteur de l'eau 80 milliards DZD (1,152 milliard \$US).

- Le compte No 302-134 : «Fonds de gestion des opérations d'investissements publics inscrites au titre du programme de consolidation de la croissance économique (PCCE) 2010-2014 " avec plus de 2000 milliards DZD pour le secteur de l'eau (soit 26,731 milliards \$US).

Il est donc aisé de comprendre pourquoi nous parlons d'un " plan Marshall de l'eau " en Algérie durant la période 1999-2012 (Figure 3). Les améliorations, bien qu’elles soient prégnantes en termes de réalisations des infrastructures et des projets, demeurent mitigées et loin d'être à la hauteur de l'effort financier consenti. En effet, les autorisations budgétaires ont évolué de manière remarquable pour atteindre un pic de 7,333 milliards \$US (535,5 milliards DZD) en 2011. La moyenne annuelle de crédits de paiement de la période (1999-2012) est de 3,117 milliards \$US. Le cumul des montants autorisés entre 1999 et 2012 a atteint 43,642 milliards \$US (soit 3 159,26 milliards DZD) tandis que les volumes réellement consommés pour la même période sont de l'ordre de 26,615 milliards \$US (1 919,02 milliards DZD) soit un taux d'absorption moyen de $60,98 \%$. Ce dernier se définit comme étant le ratio entre les crédits de paiements $(\mathrm{CP})$ et les dépenses réelles. Il s'appelle aussi le taux de consommation de crédits. Dans le cas où le ratio est inférieur à $100 \%$, il existe une sous-consommation de crédits (ou sous-absorption). Le reliquat non consommé reste endormi dans ces STA sans un contrôle budgétaire rigoureux. $\mathrm{Si}$ un sous-secteur enregistre une surconsommation, le taux dépasse $100 \%$, le surplus est payé par découvert de la banque avant qu'une demande de complément de crédits soit adressée à la direction de planification et des affaires économiques (DPAE) et ensuite à la direction générale du budget (DGB). Avec la faiblesse de la capacité d'absorption, l'effort budgétaire n'a, donc, pas fourni les résultats escomptés compte tenu de l'incapacité du secteur d'absorber tous ces fonds. Nous constatons d'ores et déjà l'existence d'une corrélation négative (le coefficient de corrélation égale à $-0,87$ ) entre les dotations financières et les taux d'absorption. Pour les premières années de la période étudiée, les taux d'absorption sont substantiels et parfois le secteur surconsomme les crédits alloués; c'est le cas de l'exercice 2002 (111\%), 2004 (115\%) et 2005 (106\%), mais au fil des ans et à partir de 2006 le taux suit une tendance baissière pour arriver à $42 \%$ en 2011 et à $39 \%$ en 2012 . Cet état explique que le secteur est suffisamment nanti en ressources financières et que la capacité d'absorption n'est pas à l'origine déterminée par les montants alloués, mais plutôt par des contraintes et des blocages institutionnels (BANQUE MONDIALE, 2007a). En pratique, plus les dotations sont importantes, plus les fonds sont sous consommés ce qui autorise à se questionner sur l'utilité de telles dotations, si aucun effort sérieux ne s'oriente vers une résolution des contraintes empêchant le bon déroulement du processus.

\section{3 Évaluation du coût économique de l'atteinte des cibles des OMD liées à l'eau potable : vision rétrospective 1999-2012}

Les améliorations qui ont été constatées dans les services de l'eau et de l'assainissement en Algérie étaient le résultat d'un effort avéré en termes de financement et de projets d'équipement et d'investissement. Ainsi, dans le cadre de cette politique largement budgétivore, des sous-secteurs qualifiés de prioritaires ont bénéficié d'enveloppes importantes dont l'AEP/adduction qui se positionne en tête durant toute la période de l'étude (entre 1999 et 2012). Dans l'objectif de déceler la place de ce segment et le coût économique de l'essor favorable des indicateurs de l'eau potable, nous mettons en exergue la répartition annuelle des crédits de paiement, des taux d'absorption et de la part du sous-secteur par rapport aux dotations budgétaires totales (Figure 4).

Le droit d'accès à l'eau et à l'assainissement est consacré par la loi No 05-12 (article 3). De ce principe découle un objectif de priorisation des besoins domestiques. Autrement dit, l'approvisionnement en eau à travers la mobilisation et la distribution de l'eau en quantité suffisante et en qualité requise se fait en priorité pour les besoins de la population et de l'abreuvement du cheptel. Pour le réaliser, l'engagement d'une politique d'investissement de grande envergure relève d'une importance colossale. La plus grande partie des dotations budgétaires allouées au secteur ont été affectées prioritairement à la mobilisation de l'eau potable et à l'extension des réseaux de distribution de l'eau. Durant la période allant de 1999 à 2012, le sous-secteur d'AEP/adduction a engrangé environ 23,459 milliards \$US du total alloué au secteur (43,642 milliards \$US) soit une part de 53,75\% du cumul autorisé. Les dotations budgétaires de l'AEP/adduction (crédits de paiement [CP]) ont augmenté de manière conséquente passant de 130,04 millions \$US en 1999 à 430,87 millions \$US en 2004 avant d'entamer l'expansion la plus notable entre 2005 (704,44 millions \$US) et 2009 (4,104 milliards \$US) (Figure 4). En 2010, une baisse 


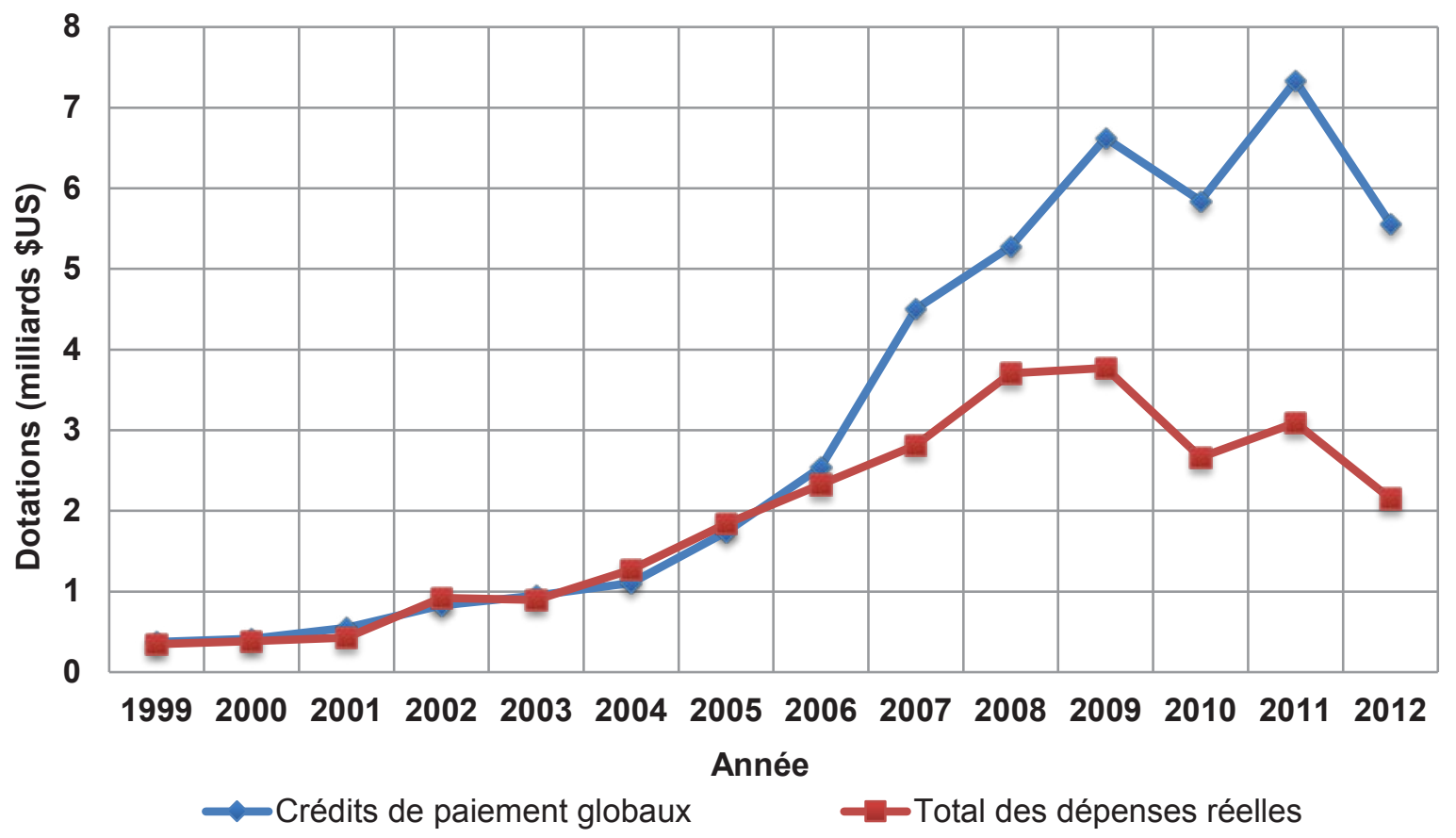

Figure 3. Dotations budgétaires et capacité d'absorption du secteur de l'eau.

Capital expenditure and absorptive capacity of the water sector in Algeria.

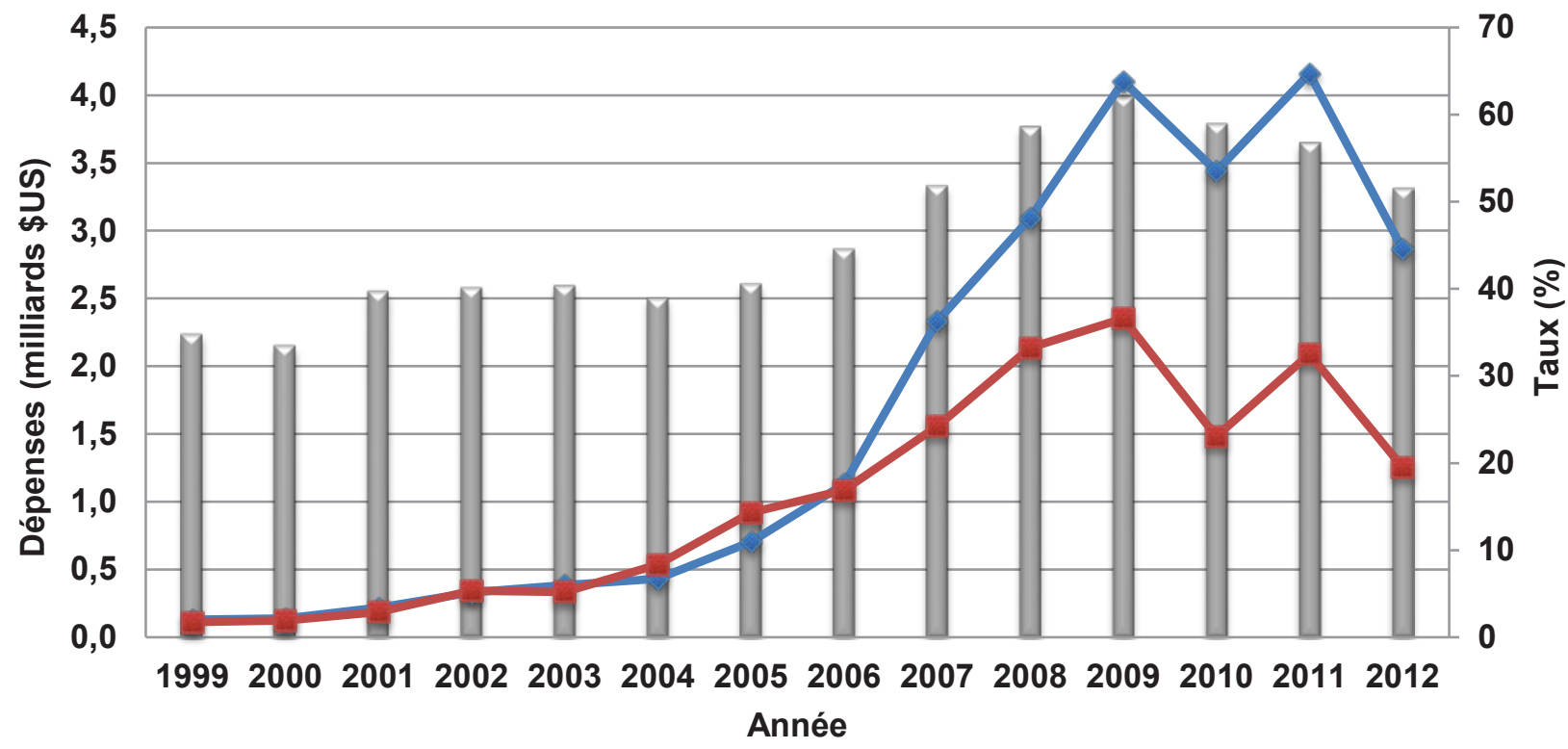

$\longrightarrow$ Part d'AEP/adduction dans les CP globaux $(\%) \longrightarrow$ CP d'AEP/adduction $—$ Dépenses réelles AEP/adduction

Figure 4. Évolution des dépenses d'équipement, du taux d'absorption et de la part des dépenses dans le sous-secteur de l'eau potable. AEP : alimentation en eau potable; $\mathrm{CP}$ : crédits de paiements.

Evolution of capital expenditure, absorption rate and expenditure share in the sub-sector of drinking water. 
des CP à un seuil de 3,44 milliards \$US a été enregistrée. Ceci s'explique par la dégringolade des cours de pétrole en 2009 à un niveau de 32,2 \$US/baril après avoir atteint le pic en 2008 avec 145,3 \$US d'où la prudence dans l'allocation du budget d'équipement. Le seuil le plus élevé a été enregistré durant l'exercice de 2011 avec un CP d'environ 4,159 milliards \$US. Toutefois, cette allocation a entamé une tendance baissière depuis en raison de la réception de certains grands projets structurants et le début d'une politique budgétaire restrictive (volet dépenses d'équipement) en Algérie.

Même si le budget d'équipement dans le sous-secteur de l'AEP/adduction a connu une nette augmentation depuis 1999 , l'absorption de ces fonds demeure modeste avec un taux d'absorption moyen estimé de $61,87 \%$ c'est-à-dire sur les 23,459 milliards \$US autorisés, le sous-secteur n'a absorbé, en fait, que 14,515 milliards \$US. En effet, le taux d'absorption entre 1999 et 2003 était aux alentours de $86 \%$ et $103 \%$. Le paroxysme a été atteint durant les deux années 2004 et 2005 avec un taux de 124,42\% et 130,64\% respectivement. Cependant, depuis, la capacité d'absorption s'est vue en baisse permanente jusqu'à un niveau de 50,24\% en 2011 et 43,83\% en 2012 (Figure 3). Ce problème de sous-consommation de crédits doit être au cœur de la réflexion sur la résolution des contraintes de la politique de l'eau potable en recherchant l'origine et les causes des écarts entre les dotations budgétaires et la réalité de décaissement dans le sous-secteur de l'AEP/adduction (voire dans tous les secteurs de l'économie). Sur le terrain, aucune institution n'a essayé de les expliquer. D'autant plus que cellesci se contentent de donner des analyses génériques qui donnent l'impression des contraintes fatales et insurmontables sans un effort particulier pour y remédier. Souvent, les raisons suivantes sont évoquées par les acteurs intervenant dans la mise en place de la politique de l'eau potable:

- la lenteur des procédures de passation de marchés, mais sans consentir des requêtes d'allégement de ces procédures de longue haleine;

- la complexité des procédures d'expropriation sans une application rigoureuse des textes réglementaires;

- l'insuffisance des crédits alloués due à la faiblesse des études de projets et des méthodes d'analyse des coûts;

- les conjonctures d'arrêt de travail en raison des intempéries et des conditions climatiques. Par exemple, les températures élevées dans le cas de transfert d'eau d'In Salah-Tamanrasset. Dans cette région du grand sud algérien, la température frôle parfois $60^{\circ} \mathrm{C}$ selon l'Office national de la météorologie. Cette température caniculaire empêche l'avancement des projets. Notons aussi que ces conjonctures d'arrêt de travail sont causées par des ruptures d'approvisionnement en matières premières bien que les grands projets sont prioritaires de par la loi;

- des interférences intrasectorielles et intersectorielles (notamment avec les secteurs du transport, de l'énergie et de l'habitat) entre les projets en dépit de l'existence des instances de coordination et de concertation chargées de la prise en charge de telles discordances.

Le sous-secteur de l'eau potable a décroché, entre 1999 et 2012, 53,75 \% du total des CP alloués au secteur de l'eau. Cette moyenne est passée de 34,74\% en 1999 à $61,97 \%$ en 2009 avant de baisser à 51,49\% en 2012. Ces indicateurs témoignent d'une attention particulière de la part des décideurs afin de dénouer la problématique d'accès à l'eau potable, mais les performances demeurent mitigées au regard du poids du financement de ce volet. De là apparaît le rôle central de la planification financière stratégique (PFS), une stratégie de financement au sens de l'OCDE (2010). La PFS repose sur l'engagement d'un processus de dialogue multipartite entre les acteurs de l'eau pour parvenir à un consensus sur le financement du secteur de l'eau et suppléer ainsi les entraves à l'amélioration de la capacité d'absorption.

\subsection{Financement de l'eau potable : vision prospective à l'horizon 2030}

Le financement de l'eau par le budget de l'État a favorisé une politique de l'offre à grande échelle surtout avec un niveau drastiquement bas pour le poste d'entretien et de maintenance. De fait, en 2010 dans tout le portefeuille géré par les établissements publics à caractère public et commercial (EPIC) et établissements publics à caractère administratif (EPA) l'entretien n'a bénéficié d'aucun sou, alors que celui géré par les DREW était égal à 0,203 million \$US sur un total alloué de 5,843 milliards \$US. À cela, il faut ajouter 0,68 million \$US sur 106,56 millions \$US alloués dans le cadre du budget de fonctionnement. En outre, les prévisions tablent sur une persistance d'une telle pratique qui privilégie l'augmentation de l'offre au lieu de jouer aussi sur la demande. Ceci se trouve bien souligné dans le cadre de la planification, notamment le PNE (2011a) : " le PNE est un projet de mobilisation de l'eau que ce soit pour le service de l'AEP ou de l'agriculture irriguée; son champ d'action est a priori celui de la production de l'eau ". Ainsi, le PNE prévoit un plan de financement de l'approvisionnement en eau potable et industrielle (AEPI) et de l'irrigation, dans la visée de satisfaire la demande à l'horizon 2030. Ce plan est susceptible à l'ajustement au gré des priorités et des contraintes budgétaires.

L'étude financière et économique du PNE (2011a) s'inscrit dans une perspective volontariste qui vise deux buts principaux à savoir la satisfaction des besoins en AEPI et l'autosuffisance alimentaire en 2030. Pour atteindre ces deux objectifs, un programme d'investissement public a été proposé avec un budget prévisionnel de 5,734 billions DZD (soit 77,889 milliards $\$ \mathrm{US})$. Ce programme est réparti entre deux sous-secteurs AEPI avec une dotation prévisionnelle de 46,574 milliards \$US (soit 
$59,8 \%$ du programme) et l'irrigation avec 31,314 milliards \$US (soit 40,2\% du programme). La primauté accordée par ce plan d'investissement à l'AEPI est inspirée du critère de priorisation des besoins domestiques dans la politique de l'eau. Le plan de financement d'investissement prévu se caractérise par :

- une moyenne d'investissement annuel d'environ : 3,894 milliards \$US (286,71335 milliards DZD) sur une période de 20 ans;

- une moyenne d'investissement annuel AEPI : 2,329 milliards \$US (171,4432 milliards DZD);

- investissement annuel AEPI par habitant : 60,174 \$US (4 430,057 DZD) (38,7 millions d'habitants en 2014).

Le tableau 1 donne un aperçu général sur l'effort budgétaire à consentir dans le futur afin de tenir le niveau d'investissement qu'il faut. À première vue, il apparait que la position de dépenses est solide et très favorable en comparant ce planning d'investissement dans l'AEPI avec la situation d'avant 2011 étant donné que les dotations budgétaires entre 2007 et 2011 étaient supérieures à la moyenne d'investissement dans l'AEPI prévue à l'horizon 2030 soit 2,329 milliards \$US. Néanmoins, l'investissement en 2012 évalué à 2,86 milliards \$US (Figure 4) était inférieur à la moyenne prévue dans le cadre du PNE (2,329 milliards \$US) ce qui montre la difficulté de maintenir une telle cadence d'investissement compliquant ipso facto les perspectives d'une continuité d'amélioration des services de l'eau en Algérie et d'une possibilité d'éradication des inégalités d'accès à l'eau entre les régions (nord $v s$ sud, rurales vs urbaines). Actuellement, la situation pourrait devenir plus précaire avec l'évolution à la baisse des cours du pétrole qui ont perdu environ $61,65 \%$ de leur valeur entre le mi-juin 2014 (112 \$US/baril) et le début d'août 2016 (42,95 \$US/baril). À cet égard, suite à la conjoncture économique peu favorable le gouvernement a décidé de geler certains projets dans l'hydraulique, et si l'instabilité des marchés pétroliers persiste dans la même tendance, l'Algérie se retrouvera, de nouveau et à moyen terme, dans le phénomène de la rareté économique de l'eau (economic water scarcity). Cet état de fait impose la nécessité impérieuse d'un programme en vue d'améliorer le financement endogène du secteur de l'eau via les trois $T$ (tarification, taxes et transferts), ainsi qu'une obligation du passage à une GDE qui favorise l'économie d'eau par le biais d'instauration de mécanismes d'incitation à la réduction de la demande. Le plan de financement décrit supra est fondé sur une hypothèse d'une demande en eau potable de $4113,1 \mathrm{hm}^{3}$ en 2030 soit un volume supplémentaire à mobiliser de 2068,9 $\mathrm{hm}^{3}$ par rapport à la demande en eau de 2010. Cet ajustement a été réalisé après avoir corrigé la demande de 2010 par rapport à la qualité de service. Le coût d'investissement pour l'AEPI durant la période 2010-2030 pourrait atteindre 46,574 milliards \$US (3 428864 millions DZD); sachant que ce chiffre est non actualisé, la prise en compte d'autres variables le rend plus conséquent qu'il apparait à première vue. Ce montant
Tableau 1. Les investissements à consentir pour l'approvisionnement en eau potable et industrielle (AEPI) et l'irrigation (20102030). (Source : PNE, 2011a)

Table 1. The planned investments in drinking and industrial water supply (AEPI) and in irrigation (2010-2030). (Data: PNE, 2011a)

\begin{tabular}{lccc}
\hline \multirow{2}{*}{ Désignation } & \multicolumn{2}{c}{ Montant de dépenses programmées } & \multirow{2}{*}{ Part (\%) } \\
\cline { 2 - 3 } & $\mathbf{1 0}^{\mathbf{6}} \mathbf{D Z D}$ & \$US & \\
\hline AEPI & 3428864 & 46574473894 & 59,8 \\
Irrigation & 2305403 & 31314432955 & 40,2 \\
Total & 5734267 & 77888906849 & 100 \\
\hline
\end{tabular}

met en lumière l'importance de l'investissement à consentir. Les montants alloués par le plan d'investissement à l'horizon 2030 montrent que la politique de l'eau en Algérie sera encore axée sur une augmentation de l'offre. En fait, 64,05\% seraient consacrés à l'adduction et au transfert d'eau; 13,49\% à la distribution de l'eau; 5,42\% aux champs captants; 9,37\% au renouvellement du patrimoine existant, $4,75 \%$ et $2,92 \%$ au dessalement de l'eau de mer et à la construction des barrages, respectivement (PNE, 2011a). Aucune mention de programme d'économie d'eau ni de politiques anti-gaspillage n'existe dans le planning. Le volet de renouvellement du patrimoine existant susceptible d'être le noyau dur d'une stratégie axée sur l'économie d'eau et de lutte contre les fuites, que ce soit des pertes dans les réseaux de distribution ou des pertes liées notamment aux raccordements illicites qui engendrent non seulement un gaspillage d'eau, mais aussi un gaspillage financier, ne représente que $9,37 \%$ de ce programme. Une part qui demeure faible vu la vétusté des infrastructures et des réseaux de distribution qui nécessitent une réhabilitation urgente. Un nombre important d'infrastructures est réalisé de manière médiocre, à la hâte et en s'appuyant sur des études de faisabilité qui manquent de maturité technique comme en témoigne la faiblesse dans l'efficience de la distribution de l'eau potable. Le plan d'investissement à l'horizon 2030 serait le garant de la réalisation des objectifs de développement durable (ODD) liés à l'eau notamment l'objectif 6 : «Garantir l'accès de tous à l'eau et à l'assainissement et assurer une gestion durable des ressources en eau " (ASSEMBLÉE GÉNÉRALE DES NATIONS UNIES, 2015). Toutefois, la mise en œuvre d'un tel programme volontariste s'avère difficile au vu de la conjoncture économique actuelle.

\subsection{Discussion des résultats}

L'Algérie a affecté des sommes colossales à la mobilisation de l'eau potable, avec un cumul d'autorisation budgétaire de 23,459 milliards $\$$ US, et à la protection des ressources en eau par le truchement du volet de l'assainissement ce qui atteste d'une période de " rattrapage hydraulique " où les retards accumulés durant les années 90 ont été relativement 
corrigés. Cette période allant de 1999 à 2012 est qualifié par nous d'une période de "rattrapage hydraulique " en Algérie vu quelques indices comme l'augmentation phénoménale des autorisations budgétaires du secteur de l'eau, une évolution favorable des raccordements aux réseaux de l'eau potable et de l'assainissement (voir supra), une augmentation des superficies équipées et irriguées ainsi la superficie agricole équipée a atteint 230000 ha en 2015 alors qu'elle était 156000 ha en 1999 (soit une augmentation de $47,43 \%$ ), le nombre de stations d'épuration des eaux usées a augmenté de 12 stations en service en 1999 à 177 en 2016, le nombre de barrages a atteint 75 barrages en exploitation au début de l'année 2016 contre 47 barrages en exploitation en 1999, une réforme institutionnelle et une réorganisation des institutions de l'eau, etc.

Si cet effort immense en termes de dépenses est indubitable, il n'en demeure pas moins que la faiblesse d'absorption de ces fonds pose un problème éminent. Le taux d'absorption moyen entre 1999 et 2012 est estimé à 61,87 \%, c'est-à-dire que les autorisations budgétaires réellement dépensées s'évaluaient alors à 14,515 milliards \$US dans le sous-secteur de l'AEP/ adduction avec une tendance baissière inquiétante $(50,24 \%$ en 2011 et $43,83 \%$ en 2012). De fait, le phénomène s'explique par des contraintes économiques, de planification, de programmation et d'exécution des projets. En plus des explications déjà évoquées, à notre sens, la capacité d'absorption dépend de l'existence d'un capital humain et d'un encadrement technique qualifié en mesure de gérer toutes ces ressources financières. Alors qu'en Algérie certaines institutions abritent des effectifs pléthoriques avec une prééminence du personnel administratif sur le personnel technique et économique. Dans le sous-secteur de l'eau potable en Algérie, le nombre de fonctionnaires pour 1000 abonnés est de 7,58 employés (ratio calculé pour l'ADE (2013) qui compte 24342 employés et 3,21 millions d'abonnés). Par conséquent, il faut améliorer qualitativement ce capital intellectuel pour relever efficacement le défi de l'eau. Sachant que dans la majorité des pays le ratio ne dépasse pas quatre employés; à Windhoek (Namibie), il est de 2 employés pour 1000 abonnés, 1,1 employés au Chili, 3 employés au Maroc, 3,5 employés en Iran (BANQUE MONDIALE, 2007a; GUMBO, 2004). A fortiori, le processus budgétaire joue un rôle prépondérant dans la détermination de la capacité d'absorption; ainsi le cycle de la budgétisation actuelle est à l'origine des retards. En fait, la procédure d'attribution des crédits est chronophage et les comptes de la fin du cycle budgétaire ne sont clôturés qu'après une période complémentaire de trois mois (BANQUE MONDIALE, 2007a). En somme, pour remédier aux contraintes qui portent atteinte à la capacité d'absorption, un travail de fond doit être réalisé pourvu qu'il intègre les critères d'efficacité, d'optimalité économique et l'obligation de résultat. Les réformes actuelles notamment le système de planification intégré (SPI) et la modernisation du système budgétaire (MSB) vont dans cette direction, mais elles n'avancent que très lentement.
Les résultats de ce travail montrent que la réalisation des cibles des OMD liées à l'eau potable, spécifiquement la cible 7.C, était coûteuse pour l'Algérie. En effet, l'atteinte des ces cibles a nécessité des dépenses réelles de 14,515 milliards \$US entre 1999 et 2012 et le coût lié aux services de l'assainissement s'estime à 5,348 milliards \$US durant la même période. Dans cette perspective, la réalisation de l'objectif 6 des ODD nécessitera un effort de financement indéniable. Le plan national de l'eau (PNE) a déjà prévu un plan de financement volontariste à l'horizon 2030 (Tableau 1) (PNE, 2011a et 2011b). Néanmoins, à l'égard des moyens déployés, les performances auraient pu (ou dû) être meilleures si les principes de la gestion intégrée des ressources en eau (GIRE) étaient bien appliqués et si les contraintes causant la faiblesse de la capacité d'absorption étaient surmontées. Quant à la cible 7.A des OMD dans son volet visant à inverser la tendance à la déperdition des ressources environnementales, la confrontation de la demande en eau de l'Algérie avec les ressources disponibles (offre naturelle ou volume exploitable) constitute un autre indicateur de la rareté en eau à savoir l'indice d'exploitation de l'eau. Ce dernier s'appelle aussi l'indice de vulnérabilité des ressources en eau (Water Ressources Vulnerability Index, WRVI). Il mesure l'ampleur de la pression exercée sur les ressources en eau. Un pays se trouve dans une situation de rareté d'eau si les prélèvements annuels par rapport au volume exploitable varient entre $20 \%$ et $40 \%$ et en situation de rareté aiguë (severely water scarce) au-delà de $40 \%$ (BROWN et MATLOCK, 2011). La démarche s'est déroulée en évaluant les prélèvements de chaque sous-secteur en commençant par la demande en eau potable qui a connu une augmentation de $150 \%$ passant de $1,25 \mathrm{~km}^{3}$ en 1999 à $3,1 \mathrm{~km}^{3}$ en 2012 , ensuite la demande en eau agricole dans les grands périmètres irrigués et les périmètres irrigués de wilayas (GPI + PIW) soit 466,5 hm en 2012, la petite et moyenne hydraulique (PMH) avec $5432,2 \mathrm{hm}^{3}$ en 2012, et enfin la demande en eau de la grande industrie estimée à $128 \mathrm{hm}^{3}$. En somme, la demande totale en $2012 \mathrm{est}$ estimée à $9126,7 \mathrm{hm}^{3}$ soit un indice d'exploitation de 80,55\% par rapport aux volumes exploitables (conventionnels et non conventionnels $\left[11,33 \mathrm{~km}^{3}\right]$ ) et $87,17 \%$ par rapport au volume exploitable des ressources conventionnelles $\left(10,47 \mathrm{~km}^{3}\right)$ (KHERBACHE, 2013 et 2014a).

\section{CONCLUSION}

L'estimation du coût économique de la réalisation des cibles des OMD liées à l'eau potable requiert, d'une part, un accès à l'information économique qui reflète l'ensemble des dépenses engagées en vue d'une amélioration du service d'AEP et, d'autre part, une disponibilité de l'information technique qui montre l'état réel du service et susceptible de dévoiler les inégalités d'accès à la ressource, et ce à travers des enquêtes de 
terrain auprès des usagers. Or, l'Algérie se trouve actuellement dans une situation de déficit d'information et de transparence (information gap) au sens de l'OCDE (2012) et CHARBIT (2011) où l'accès à ces données est délicat. En dépit de ces faiblesses, ce papier a visé, autant que possible, à souligner l'effort de financement et d'investissement dans le sous-secteur de l'AEP/adduction, les progressions constatées durant la période 1999-2012 et les contraintes gangrénant ce segment. Il y a lieu de rappeler que cet essai d'évaluation du coût économique de la réalisation des cibles des OMD liées à l'eau potable en Algérie n'est pas exhaustif puisque l'étude se limite à l'évaluation des PSC et de PSD gérés par le MREE alors que certains portefeuilles liés directement au secteur de l'eau ne sont pas gérés par le MREE comme les PCD, qui relèvent de la compétence du ministère de l'intérieur et des collectivités locales, et la réalisation des stations de dessalement. Pour ce dernier volet, la promotion, la gestion et la production de l'eau dessalée sont assurées par la société Algerian Energy Company (AEC) filiale de la Sonatrach et de la Sonelgaz, sous l'autorité du ministère de l'Énergie et des Mines (MEM). Ensuite l'eau sera vendue à l'ADE. Au niveau du MREE une commission de l'eau dessalée assure le processus de vente.

Les projets de mobilisation de l'eau en Algérie ont accentué la pression sur les ressources en eau. Cette évolution suivra, sans doute, la même tendance dans le cadre du programme d'investissement à l'horizon 2030. En 1999, l'investissement par tête dans l'AEP/adduction était 4,34 \$US avant de toucher la barre de 116,36 \$US lors de l'exercice de 2009. Celui-ci a atteint le niveau de 77,21 \$US en 2012. Dans la cadre du planning d'investissement à l'horizon 2030 le ratio devrait être 60,174 \$US (avec une population de 38,7 millions d'habitants en 2014). Il est donc évident que le maintien de la cadence d'investissement de la période 1999-2012 serait très difficile d'autant plus que le nombre de la population ira en augmentation perpétuelle avec une prévision d'une population dépassant 50,7 millions d'habitants à l'horizon 2030. Par conséquent, cette option d'investissement volontariste comme pierre angulaire de la politique de l'eau doit s'accompagner par des actions effectives et concrètes sur le terrain comme une valorisation des ressources déjà mobilisées par une GDE, une contribution effective de l'usager dans le coût réel de l'eau par l'application du principe de péréquation sociale dans le système de tarification, une application des standards de gestion efficace en assurant une transparence de la planification budgétaire et une maitrise de l'information technique et économique afin de relever le défi des objectifs du développement durable (ODD). Ces éléments sont les garants d'une décision efficiente et d'une rentabilité socio-économique des projets à réaliser d'autant plus que les indicateurs de financement de l'eau en Algérie sont volatiles et fortement corrélés avec une variable exogène à savoir les cours du pétrole.

\section{RÉFÉRENCES BIBLIOGRAPHIQUES}

ASSEMBLÉE GÉNÉRALE DES NATIONS UNIES (2000). Déclaration du Millénaire. Résolution adoptée par l'Assemblée générale de l'ONU le 13 septembre 2000, ONU, New York, NY, États-Unis.

ASSEMBLÉE GÉNÉRALE DES NATIONS UNIES (2015). Transformer notre monde : le programme de développement à l'horizon 2030. Résolution adoptée le 25 septembre 2015, ONU, New York, NY, États-Unis.

BANQUE MONDIALE (2007a). RADP à la recherche d'un investissement public de qualité : revue des dépenses publiques. Rapport No 36270-DZ, Washington, DC, États-Unis, Vol. 1, 234 p.

BANQUE MONDIALE (2007b). RADP à la recherche d'un investissement public de qualité : revue des dépenses publiques. Rapport No 36270-DZ, Washington, DC, États-Unis, Vol. 2, 157 p.

BHAGWATI, J. (2010). Repenser les OMD. Financ. Dev., 47 (3), 14-16.

BROWN, A. et M.D. MATLOCK (2011). A review of water scarcity indices and methodologies. Sustainability Consortium, Food, Beverage \& Agriculture, University of Arkansas, White paper 106, 21 p.

CHARBIT, C. (2011). Governance of public policies in decentralised contexts: The multi-level approach. OCDE Regional Development Working Papers, Paris, France, $23 \mathrm{p}$.

CONSEIL NATIONAL ÉCONOMIQUE ET SOCIAL (CNES) (2000). L'eau en Algérie : le grand défi de demain. Commission de l'Aménagement du territoire et de l'Environnement, Alger, Algérie, 84 p.

DIRECTION DE LA PROGRAMMATION ET LE SUIVI BUDGÉTAIRES (DPSB) (2012). Annuaire statistique de la wilaya de Bejaia. DPSB, Algérie, 200 p.

KERTOUS, M. (2010). La gestion de l'eau potable en Algérie, quelle stratégie face aux contraintes économiques et climatiques? Ge colloque international : Stratégies de développement: Quel chemin parcouru? Quelles réponses face aux nouvelles contraintes économiques et climatiques?, 21-23 juin, Hammamet, Tunisie, Compte rendu, 21 p. 
KHERBACHE, N. (2013). Vers une nouvelle perception de la rareté des ressources en eau : Essai d'application des indicateurs de la rareté au cas de l'Algérie. $2^{e}$ colloque international sur la gestion intégrée des ressources en eau, 22-23 octobre, Batna, Algérie, Compte rendu,12 p.

KHERBACHE, N. (2014a). La problématique de l'eau en Algérie : enjeux et contraintes. Mémoire de magistère, Univ. Bejaia, Algérie, 266 p.

KHERBACHE, N. (2014b). Le financement de l'eau en Algérie : comment concilier entre équité sociale, efficience économique et responsabilisation institutionnelle? Colloque international: Système financier et croissance économique, 6-7 mai, École Supérieure de Commerce d’Alger, Algérie, Compte rendu, $18 \mathrm{p}$.

GUMBO, B. (2004). The status of water demand management in selected cities of southern Africa. Phys. Chem. Earth, 29, 1225-1231.

HADJI,T. (2005). Eau potable et irrigation : tensions et solutions en matière de financement. Dans : Le prix de l'avenir : le développement durable en Algérie. BENACHENHOU, A. (Éditeur), Thotm éditions, Paris, France, pp. 46-71.

MINISTÈRE DES RESSOURCES EN EAU (MRE) (2008). Bilan et perspective. Document du MRE du 18 mai, Alger, Algérie, $25 \mathrm{p}$.

MINISTĖRE DES RESSOURCES EN EAU (MRE) (2011). Services de l'eau en Algérie : Faire du droit à l'eau une réalité pour tous. Communication à la consultation des acteurs étatiques sur les bonnes pratiques dans les domaines de l'eau et de l'assainissement, Genève, Suisse, 28 p.

MINISTÈRE DES RESSOURCES EN EAU - GESTION INTÉGRéE DES RESSOURCES EN EAU (MRE-GIZ) (2011). Manuel-guide de planification intégrée de l'eau en Algérie : Système de planification intégrée (SPI). Projet de coopération MRE association Allemande (GIZ), 271 p.

ORGANISATION DE COOPÉRATION ET DE DÉVELOPPEMENT ÉCONOMIQUES (OCDE) (2010). De l'eau pour tous: Perspectives de l'OCDE sur la tarification et le financement. Édition OCDE, Paris, France, $155 \mathrm{p}$.
ORGANISATION DE COOPÉRATION ET DE DÉVELOPPEMENT ÉCONOMIQUES (OCDE) (2012). La gouvernance de l'eau dans les pays de l'OCDE: une approche pluri-niveaux. Édition OCDE, Paris, France, $275 \mathrm{p}$.

ORGANISATION DES NATIONS UNIES (ONU) (2005). Rapport national sur les OMD : Algérie. Rapport établi par le gouvernement algérien. OMD-DZ, Algérie, 84 p.

ORGANISATION DES NATIONS UNIES (ONU) (2011). Objectifs du Millénaire pour le développement. Rapport 2011, ONU, New York, NY, États-Unis, 72 p.

ORGANISATION DES NATIONS UNIES (ONU) (2014a). Evaluation des progrès accomplis en Afrique dans la réalisation des objectifs du Millénaire pour le développement: Analyse de la Position commune africaine sur le programme de développement pour l'après-2015. Rapport OMD 2014, ONU, Commission économique pour l'Afrique, AddisAbeba, Éthiopie, 178 p.

ORGANISATION DES NATIONS UNIES (ONU) (2014b). Objectifs du Millénaire pour le développement. Rapport 2014, ONU, New York, NY, États-Unis, 59 p.

PLAN NATIONAL DE L'EAU (PNE) (2011a). La politique nationale de l'eau. Réalisation de l'étude d'actualisation du Plan National de l'Eau. Groupement SOFRECOGrontmij/Carl-Bro-Progress-OIEau, 87 p.

PLAN NATIONAL DE L'EAU (PNE) (2011b). Adéquation des ressources et demandes. Réalisation de l'étude d'actualisation $d u$ PNE. Groupement SOFRECO-Grontmij/Carl-BroProgress-OIEau, 207 p.

RIJSBERMAN, F. (2006). Water scarcity: Fact or fiction. Agric. Water Manage., 80, (1-3), 5-22.

TURTON, A. (2002). Water demand management (WDM), natural resource reconstruction and adaptive capacity: Establishing the linkage between variables, WARFSA Research Project PJ02/99, 85 p.

UNITED NATIONS WORLD WATER DEVELOPMENT REPORT 4 (WWDR4) (2012). Managing water under uncertainty and risk. WWAP-UNESCO Report, United Nations Educational, Scientific and Cultural Organization, Paris, France, Vol. 1, 407 p. 